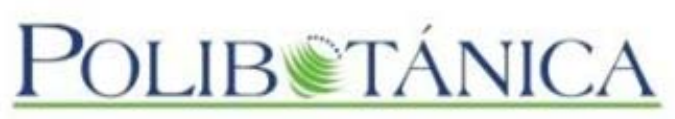

\title{
Polibotánica
}

ISSN electrónico: 2395-9525

polibotanica@gmail.com

Instituto Politécnico Nacional

México

http:www.polibotanica.mx

\section{ANÁLISIS PALINOLÓGICO DE MIELES DE Apis mellifera L. (APIDAE), ESTADO DE MORELOS, MÉXICO*}

\section{PALYNOLOGICAL ANALYSIS OF HONEY FROM Apis mellifera L. (APIDAE), STATE OF MORELOS, MEXICO*}

\begin{abstract}
Vázquez-Fuentes, Y.G.; D.L. Quiroz-García**, J.S. Acosta-Castellanos, y R. Fernández-Nava** ANÁLISIS PALINOLÓGICO DE MIELES DE Apis mellifera L. (APIDAE), ESTADO DE MORELOS, MÉXICO*.

PALYNOLOGICAL ANALYSIS OF HONEY FROM Apis mellifera L. (APIDAE), STATE OF MORELOS, MEXICO*.

*Proyecto apoyado por la Secretaría de Investigación y Posgrado del Instituto Politécnico Nacional, Clave 20100574. ** Becarios de la Comisión de Operaciones y Fomento de Actividades Académicas del IPN.
\end{abstract}

\section{POLIBETANICA}

Instituto Politécnico Nacional
Núm. 48: 87-98 México. Julio 2019

DOI: $10.18387 /$ polibotanica.48.7 (c) (i) (2) 


\section{ANÁLISIS PALINOLÓGICO DE MIELES DE Apis mellifera L. (APIDAE),} ESTADO DE MORELOS, MÉXICO

\section{PALYNOLOGICAL ANALYSIS OF HONEY FROM Apis mellifera L. (APIDAE), STATE OF MORELOS, MEXICO}

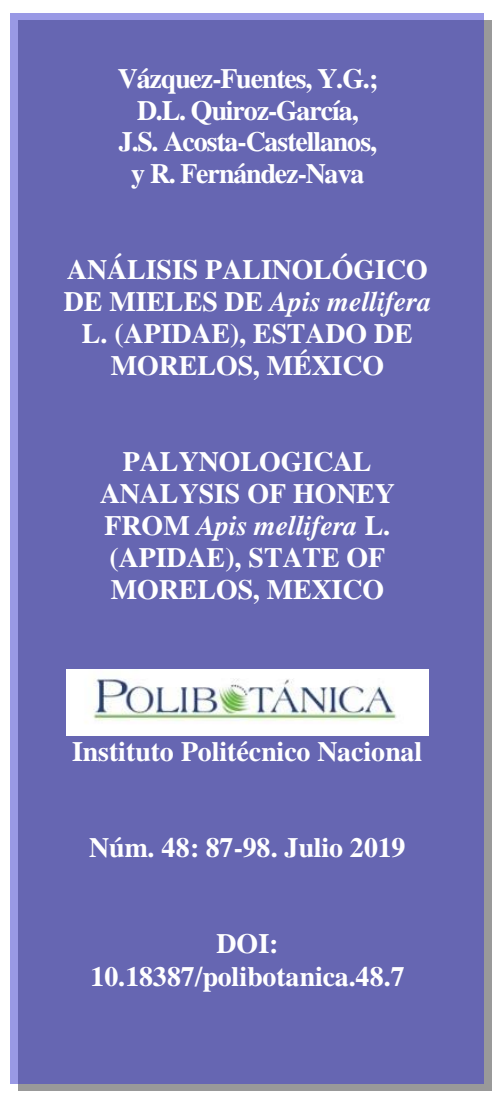

\author{
Y.G. Vázquez-Fuentes \\ D.L. Quiroz-García / dlquirozgar@yahoo.com.mx \\ J.S. Acosta-Castellanos \\ R. Fernández-Nava \\ Departamento de Botánica \\ Escuela Nacional de Ciencias Biológicas, IPN. \\ Prolongación de Carpio y Plan de Ayala, Col. Santo Tomás. \\ CP 11340 México, DF.
}

RESUMEN: Se realizó el análisis palinológico de tres muestras de miel de Apis mellifera provenientes de tres localidades del estado de Morelos. Las muestras se procesaron siguiendo la técnica de acetolólisis de Erdtman levemente modificada. Se identificaron 23 tipos polínicos pertenecientes a 18 familias, de los cuales ocho presentan porcentajes mayores al $10 \%$ a mencionar: Arracacia sp. (Apiaceae); Asteraceae tipo 1 y tipo 2; Brassica sp. y tipo 1 (Brassicaceae); ChenopodiaceaeAmaranthaceae; Salvia sp. (Lamiaceae) y Lopezia sp. (Onagraceae). Las familias mejor representadas fueron: Asteraceae y Brassicaceae. Plantas arvenses y ruderales del estrato herbáceo fueron las más importantes. Se determinó que dos de las mieles analizadas fueron monoflorales y una fue multifloral.

Palabras clave: Melisopalinología, caracterización de miel, miel monofloral, miel multifloral.

ABSTRACT: The analysis palynological of three samples of honey produced by Apis mellifera in three localities of the State of Morelos was made. The samples were processed according to the technique of Erdtman's acetolysis slightly modified. We identified 23 pollen types from 18 families, of which eight are percentages higher than 10\% mention: Arracacia sp. (Apiaceae); Asteraceae type 1 and type 2; Brassica sp. and type 1 (Brassicaceae); Chenopodiaceae-Amaranthaceae; Salvia sp. (Lamiaceae) and Lopezia sp. (Onagraceae). The best represented families were: Asteraceae and Brassicaceae. Weed plants and ruderal herbaceous stratum were the most important. It was determined that two of the analyzed honeys were monofloral and one was multifloral.

Key words: Melissopalynology, honey characterization, monofloral honey, multifloral honey.

\section{INTRODUCCIÓN}

México es el sexto productor mundial de miel y el tercer exportador a escala global (Contreras-Escareño, y otros, 2013), sin embargo, el conocimiento del origen botánico y geográfico de las mieles está basado exclusivamente en observaciones apibotánicas, en el momento en que las abejas pecorean para colectar el néctar y/o los granos de polen, éste método empírico dificulta mucho la validación de que alguna miel pueda provenir de una planta o cultivo específico, así como de que proceda de una región 
determinada (Carmona, 1980). Por consecuencia es importante determinar el tipo de polen presente en la miel, mediante el análisis melisopalinológico, así como relacionarlo con la vegetación en los alrededores del apiario, lo que contribuye a verificar la autenticidad de las mieles en cuanto a su origen botánico, así como de su origen geográfico (Ramírez \& Montenegro, 2004) (Córdova-Córdova, Ramírez-Arriaga, Martínez-Hernández, \& ZaldivarCruz, 2013).

Entre las investigaciones apícolas y melisopalinológicos realizadas en México se cuenta con los trabajos de (Ordetx, Zosaya-Rubio, \& Franco-Millan, 1972) que contribuyeron al conocimiento de la flora apícola a nivel nacional, mencionando las regiones más propicias para la apicultura en México y las plantas de mayor utilidad para las abejas. (Acosta-Castellanos \& PalaciosChávez, Proceedings of the IX International Palynological Congress, 2001) analizaron dos muestras de miel y cargas de polen procedentes de dos apiarios en la zona de Pluma Hidalgo, Oaxaca durante un ciclo anual. (Piedras-Gutiérrez \& Quiroz-García, 2007) analizaron dos muestras de miel de la zona sur del Valle de México, determinaron 19 tipos polínicos de los cuales mencionan a cuatro como de importancia. (Castellanos-Potenciano, Ramírez-Arriaga, \& Zaldivar-Cruz, 2012) determinaron en muestras de miel del estado de Tabasco, 29 tipos polínicos que consideraron como importantes y que corresponden a la flora nativa, la mayoría de las mieles analizadas fueron multiflorales, caracterizando a otras como monoflorales o biflorales. (Córdova-Córdova, Ramírez-Arriaga, Martínez-Hernández, \& Zaldivar-Cruz, 2013) analizaron mieles de Tabasco y no encontraron diferencias entre ellas en cuanto a sus características fisicoquímicas, mientras que desde el punto de vista palinológico encontraron mieles monoflorales, biflorales y poliflorales. (Alaniz-Gutiérrez, y otros, 2017) determinaron que el $65 \%$ de las mieles producidas en el Valle de Mexicali, Baja California son monoflorales $\mathrm{y}$ encontraron diferencias entre las cosechas de un mismo apiario.

El estado de Morelos forma parte de la región apícola del altiplano y aunque sólo aporta el $2 \%$ de la producción nacional de miel, la apicultura es una de las actividades de gran importancia en la entidad, con amplio arraigo en la población y que se practica en todo el estado, en condiciones climáticas variadas (Vélez, Espinoza, Amaro, \& Arechavaleta, 2016). Sin embargo, son pocos los trabajos que aportan información para el desarrollo de la apicultura en la entidad, solo se cuenta con algunas descripciones de especies melíferas en diferentes tipos de vegetación (Carmona, 1980) y el estudio palinológico de mieles producidas por Apis mellifera en tres municipios del estado de Morelos (Cuernavaca, Tepoztlán y Tlayacapan), en donde se determinaron mieles multiflorales y monoflorales (Quiroz-García \& Arreguín-Sánchez, 2008), por lo que para dar continuidad con estos trabajos, el presente estudio tiene como propósito determinar los principales recursos botánicos utilizados por Apis mellifera por medio del análisis palinológico de muestras de miel de tres localidades del estado de Morelos, lo que permitirá caracterizar las mieles y darles un valor agregado para su comercialización.

\section{MATERIAL Y MÉTODOS}

El estado de Morelos se localiza en la parte central del país, en la vertiente del sur de la serranía del Ajusco y dentro de la cuenca del río Balsas, está dividido en municipios entre los que se encuentran Cuautla, Tlalnepantla y Totolapan (fig. 1), entidades de donde se compraron las muestras de miel analizadas. En Cuautla ( $18^{\circ} 49^{\prime}$ de latitud norte y $99^{\circ} 01^{\prime}$ de longitud oeste) el clima predominante es cálido subhúmedo con lluvias en verano, la altitud promedio es de 1300 $\mathrm{msnm}$, la temperatura promedio anual es de $20.5^{\circ} \mathrm{C}$ y la precipitación oscila entre $800-1000$ $\mathrm{mm}$; la vegetación de la zona es bosque tropical caducifolio en diferente grado de deterioro, asociado a pastizales y amplias áreas de agricultura de riego.

En el municipio de Tlalnepantla ( $18^{\circ} 57^{\prime}$ de latitud norte y los $98^{\circ} 14^{\prime}$ de longitud oeste) el clima es templado subhúmedo, con una altitud promedio de $2060 \mathrm{msnm}$, la temperatura promedio 
anual es de $17^{\circ} \mathrm{C}$ y la precipitación promedio anual de $2341 \mathrm{~mm}$; en el área se tienen bosques de pino y encino, circundados de zonas agrícolas.

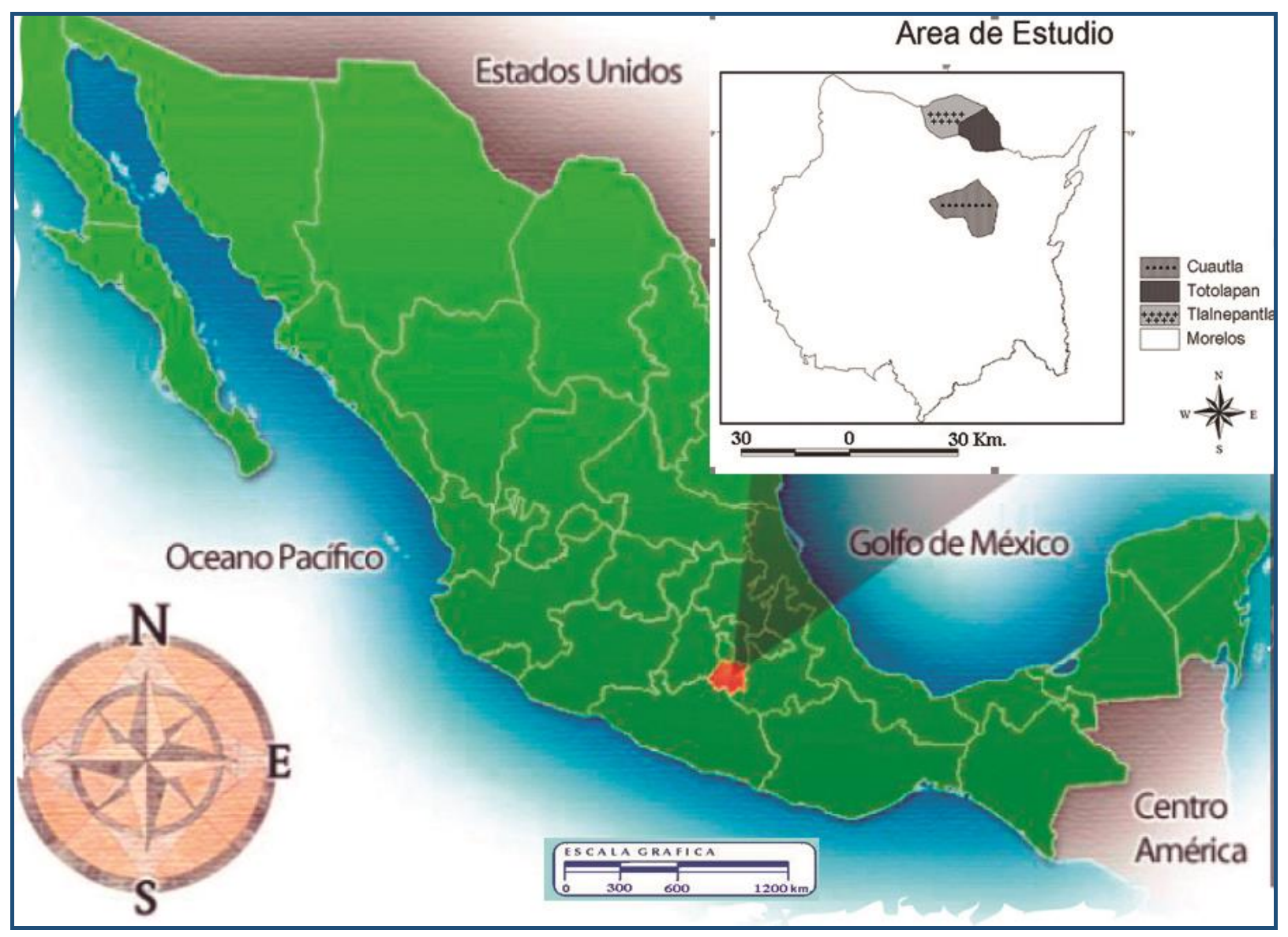

Fig. 1. Ubicación del área de estudio.

El municipio de Totolapan ( $18^{\circ} 58^{\prime}$ de latitud norte y los $98^{\circ} 55^{\prime}$ de longitud oeste) tiene un clima subtropical húmedo, la altitud es de $1897 \mathrm{msnm}$, la temperatura media anual es de 17.4 ${ }^{\circ} \mathrm{C}$ y la precipitación media anual es de $913 \mathrm{~mm}$. La vegetación se caracteriza por bosque tropical caducifolio y zonas agrícolas.

(http://www.inafed.gob.mx/work/enciclopedia/EMM17morelos/municipios/17006a.html).

La muestra de Cuautla se obtuvo en el mes de octubre del 2004, la de Tlalnepantla en agosto del 2006 y la de Totolapan en octubre del 2008. De cada muestra se tomaron $10 \mathrm{~g}$ y se diluyeron en agua acidulada caliente, posteriormente se procesaron de acuerdo a la técnica de acetólisis (Erdtman, 1952) (Lieux, 1980) modificando los tiempos de centrifugación y manteniendo las muestras durante 20 minutos en la mezcla acetolítica (anhídrido acético y ácido sulfúrico en proporción 9:1) a baño maría a temperatura entre 70 y $80{ }^{\circ} \mathrm{C}$; se prepararon tres laminillas permanentes con gelatina glicerinada sin colorante y con fucsina básica, por cada muestra para su análisis al microscopio de luz para su identificación y cuantificación.

La identificación se realizó utilizando claves palinológicas (Palacios, 1968) (Roubik \& Moreno, 1991) (Palacios, Ludlow-Wiechers, \& Villanueva, 1991) y por comparación con la colección de 
referencia del estado de Morelos depositada en la Palinoteca de la Escuela Nacional de Ciencias Biológicas del Instituto Politécnico Nacional, se tomaron microfotografías de los tipos polínicos más frecuentes.

Para obtener las frecuencias porcentuales de cada tipo polínico observado se realizó un conteo de 500 granos de polen por muestra (Acosta-Castellanos, Quiroz-García, Arreguín-Sánchez, \& Fernández-Nava, 2011). Los taxones identificados se clasificaron de acuerdo a las categorías propuestas por (Loveaux, Mauricio, \& Vorwohl, 1978) como polen dominante $(\geq 45 \%)$, polen secundario (del 16 al $45 \%$ ), polen de importancia menor ( 3 al $15 \%$ ) y polen menor $(\leq 3 \%)$. Las mieles se designarán como monoflorales cuando un tipo de polen presente un porcentaje mayor o igual a $45 \%$ del total de los tipos polínicos encontrados y multiflorales o poliflorales cuando más de un tipo polínico alcance un porcentaje igual o mayor al 10\% (Lieux, 1972). Se consideraron como las especies más importantes como fuentes de néctar/polen las que tuvieron una representación del $10 \%$ o superior de acuerdo con (Ramalho, Imperatriz-Fonseca, KleinertGiovannini, \& Cortopassi Laurino, 1985), mientras que los taxones presentes en bajos porcentajes son considerados recursos alimentarios alternativos (Cortapassi-Laurino \& Ramalho, 1988).

\section{RESUlTADOS}

Se identificaron 23 tipos polínicos pertenecientes a 18 familias. La tabla 1 muestra las abundancias relativas de cada uno de los tipos polínicos encontrados en las diferentes muestras. Ocho de ellos se consideran de importancia por presentar porcentajes mayores al 10\% (fig. 6).

En la muestra de miel del municipio de Cuautla, se determinó el polen de 11 tipos polínicos, los más abundantes fueron: Chenopodiaceae-Amaranthaceae (48.3\%), Asteraceae Tipo 2 (21.3\%) y Arracacia (10.3\%) (figs. 2 y 3 ).

En la muestra de Tlalnepantla se encontró polen de 10, los más abundantes fueron: Asteraceae Tipo 1 (45.2\%), Salvia (20.4\%), Lopezia (15.5\%) y Asteraceae Tipo 2 (10.5\%) (figs. 2 y 4).

En la muestra del municipio de Totolapan se determinaron 16, los más frecuentes fueron: Brassica (23.4\%), Chenopodiaceae-Amaranthaceae (19.4\%) y Brassicaceae Tipo 1 (17.8\%) (figs. 2 y 5 ).

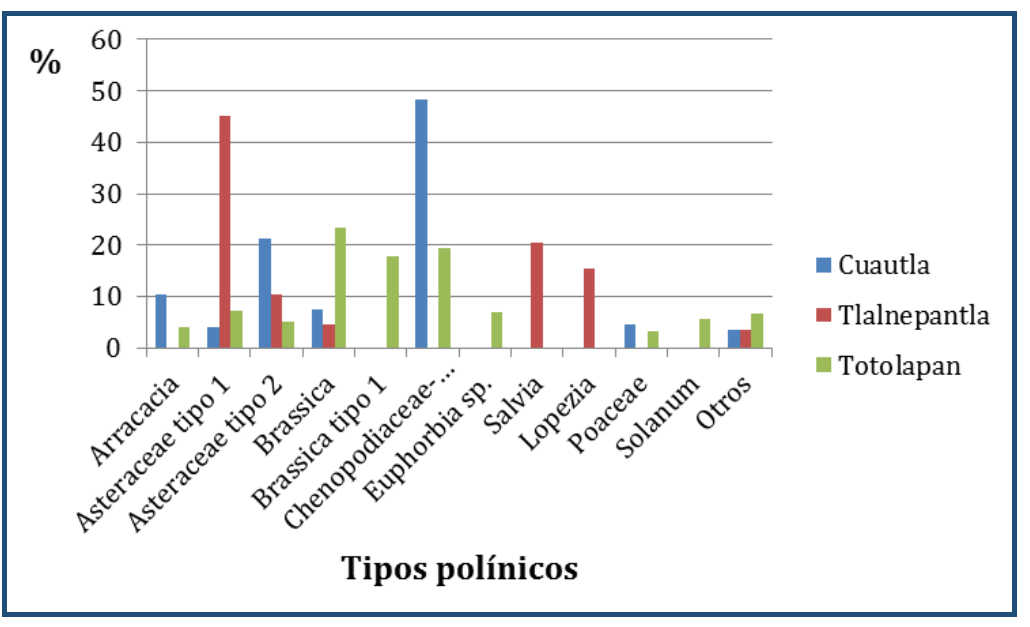

Fig. 2. Frecuencia relativa de especies con porcentajes $>3 \%$ encontradas en las mieles de Morelos. 
Los taxones comunes a las tres muestras analizadas fueron: Taraxacum officinale Weber, Asteraceae Tipo 1, Asteraceae Tipo 2 y Brassica sp. Las familias con mayor diversidad de tipos polínicos representados en las muestras fueron: Asteraceae y Brassicaceae. Al considerar las clases de frecuencias, las familias más importantes para el municipio de Cuautla fueron Chenopodiaceae-Amaranthaceae, Asteraceae y Apiaceae; en Tlalnepantla, las Asteraceae, Lamiaceae y Onagraceae; mientras que en Totolapan las familias importantes fueron Brassicaceae, Chenopodiaceae-Amaranthaceae y Asteraceae.

De las tres muestras de miel analizadas, las de Cuautla y Tlalnepantla corresponden a mieles monoflorales, con el $48.3 \%$ de polen de Chenopodiaceae-Amaranthaceae en la primera y $45.2 \%$ de Asteraceae Tipo 1 en la segunda. Sólo la muestra de Totolapan corresponde a miel multifloral debido a que ningún tipo polínico fue dominante.

El estrato mejor representado en las mieles analizadas fue el herbáceo seguido por el arbustivo y arbóreo.

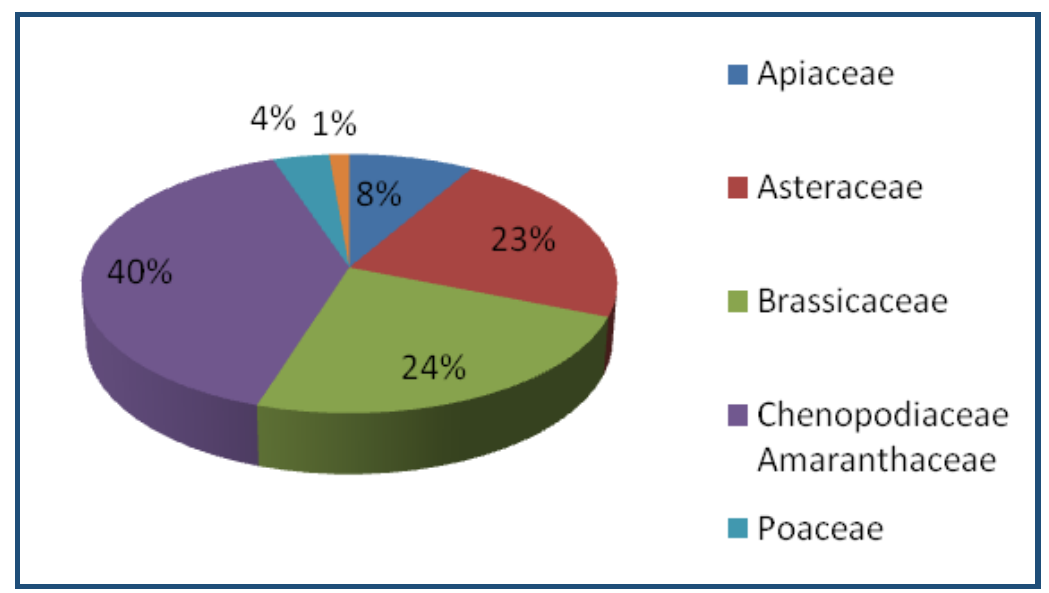

Fig. 3. Diagrama de frecuencias de aparición de las familias presentes en la muestra de miel del municipio de Cuautla, Morelos.

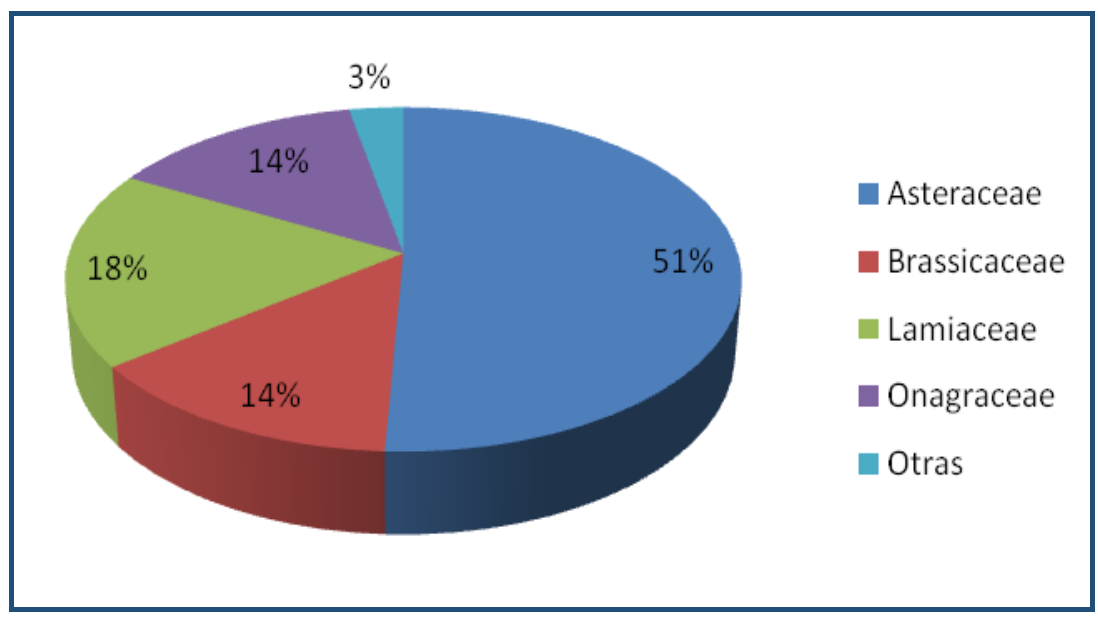

Fig. 4. Diagrama de frecuencias de aparición de las familias presentes en la muestra de miel del municipio de Tlalnepantla, Morelos. 
Tabla 1. Porcentaje de los tipos polínicos presentes en las muestras de miel de 3 municipios del estado de Morelos. Polen dominante (D: $\geq 45 \%$ ); polen secundario (S: 16-45\%); polen de importancia menor (I: 3-15\%); polen menor $(\mathrm{m}: \leq 3 \%)$. * Polen anemófilo.

\begin{tabular}{|c|c|c|c|}
\hline Tipos polínicos & Cuautla & Tlalnepantla & Totolapan \\
\hline \multicolumn{4}{|l|}{ Apiaceae } \\
\hline Arracacia sp. & $10.33(\mathrm{I})$ & & $3.96(\mathrm{I})$ \\
\hline Apocynaceae & $0.67(\mathrm{~m})$ & & \\
\hline \multicolumn{4}{|l|}{ Asteraceae } \\
\hline Taraxacum officinale Weber & $0.33(\mathrm{~m})$ & $0.33(\mathrm{~m})$ & $1.32(\mathrm{~m})$ \\
\hline Tipo Ambrosia & $1.67(\mathrm{~m})$ & & $0.33(\mathrm{~m})$ \\
\hline Tipo 1 & $4(\mathrm{I})$ & $45.21(\mathrm{D})$ & $7.26(\mathrm{I})$ \\
\hline Tipo 2 & $21.33(\mathrm{~S})$ & $10.56(\mathrm{I})$ & $5.28(\mathrm{I})$ \\
\hline \multicolumn{4}{|l|}{ Brassicaceae } \\
\hline Brassica sp. & $7.67(\mathrm{I})$ & $4.62(\mathrm{I})$ & $23.43(\mathrm{~S})$ \\
\hline Tipo 1 & & & $17.82(\mathrm{~S})$ \\
\hline Bignoniaceae & & $2.31(\mathrm{~m})$ & \\
\hline \multicolumn{4}{|l|}{ Betulaceae } \\
\hline Alnus sp. * & & $0.33(\mathrm{~m})$ & \\
\hline Cactaceae & & & $0.33(\mathrm{~m})$ \\
\hline Chenopodiaceae-Amaranthaceae* & $48.33(\mathrm{D})$ & & $19.47(\mathrm{~S})$ \\
\hline \multicolumn{4}{|l|}{ Euphorbiaceae } \\
\hline Euphorbia sp. & & & $6.93(\mathrm{I})$ \\
\hline Fabaceae & & & $1.32(\mathrm{~m})$ \\
\hline Papilionoideae & $0.33(\mathrm{~m})$ & & $1.98(\mathrm{~m})$ \\
\hline \multicolumn{4}{|l|}{ Lamiaceae } \\
\hline Salvia sp. & & $20.46(S)$ & \\
\hline Malpighiaceae & & & $0.33(\mathrm{~m})$ \\
\hline \multicolumn{4}{|l|}{ Onagraceae } \\
\hline Lopezia sp. & & $15.51(\mathrm{~S})$ & \\
\hline \multicolumn{4}{|l|}{ Papaveraceae } \\
\hline Bocconia arborea S. Wats. & & $0.33(\mathrm{~m})$ & \\
\hline \multicolumn{4}{|l|}{ Pinaceae } \\
\hline Pinus sp.* & & $0.33(\mathrm{~m})$ & \\
\hline Poaceae* & $4.67(\mathrm{I})$ & & $3.30(\mathrm{I})$ \\
\hline \multicolumn{4}{|l|}{ Solanaceae } \\
\hline Solanum sp. & $0.67(\mathrm{~m})$ & & $5.61(\mathrm{I})$ \\
\hline Urticaceae* & & & $1.32(\mathrm{~m})$ \\
\hline Número tipos polínicos/muestra & 11 & 10 & 16 \\
\hline Tipos polínicos $\geq 10 \%$ & 3 & 4 & 3 \\
\hline
\end{tabular}




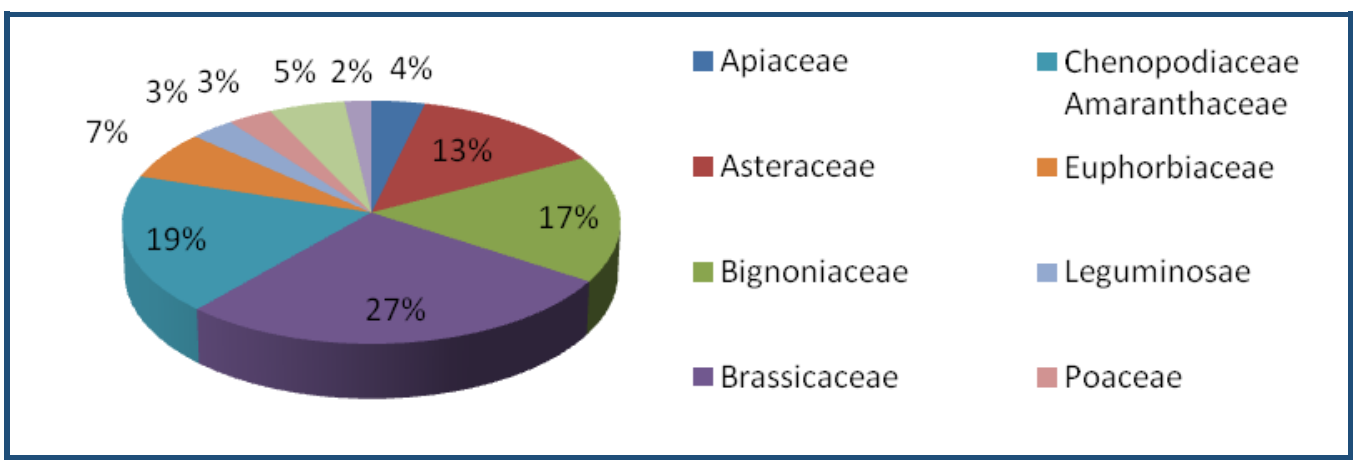

Fig. 5. Diagrama de frecuencias de aparición de las familias presentes en la muestra de miel del municipio de Totolapan, Morelos.

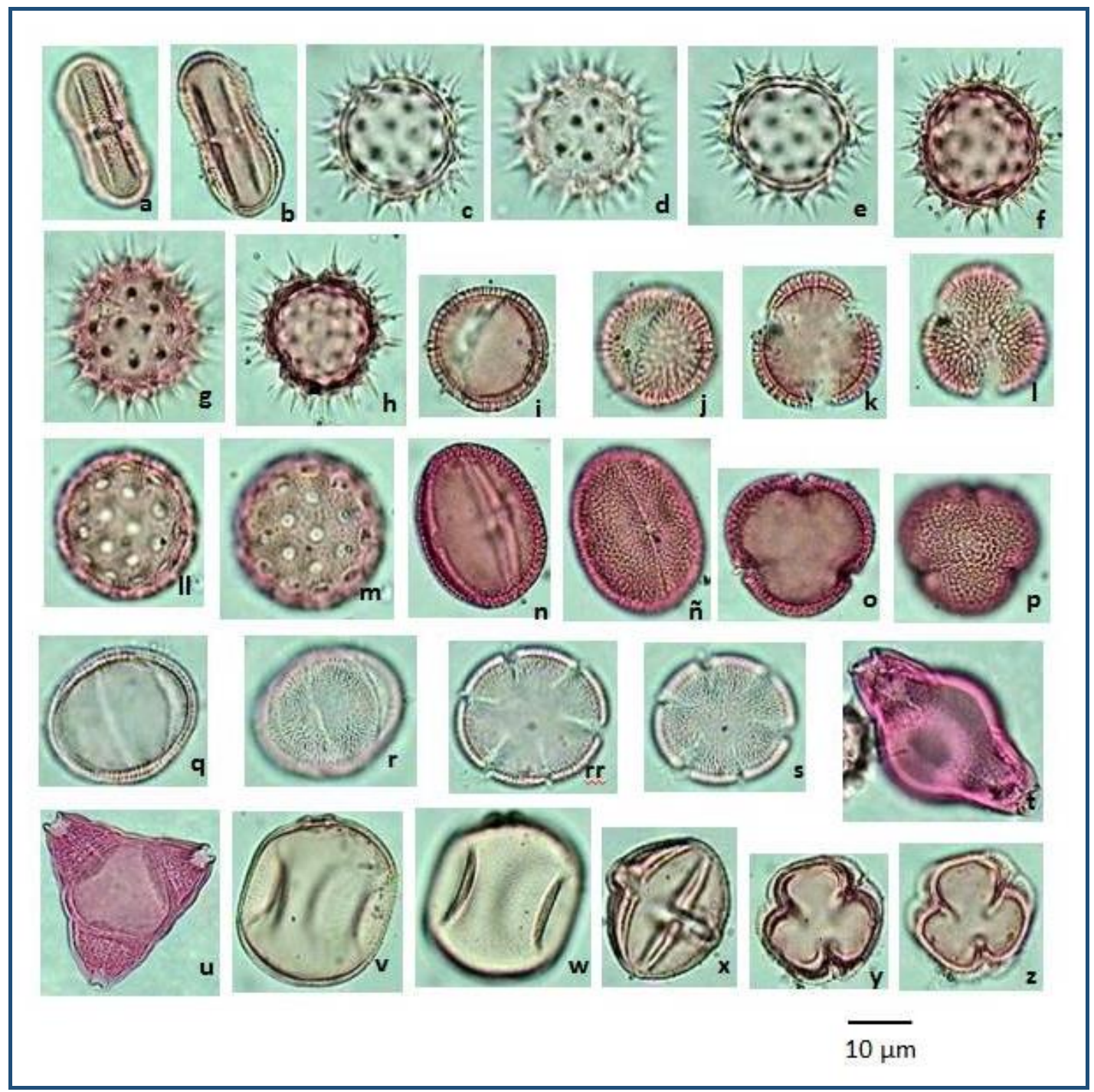

Fig. 6. a-b. Arracacia; c-e. Asteraceae Tipo 1; f-h. Asteraceae Tipo 2; i-1. Brassica; 11-m. ChenopodiaceaeAmaranthaceae; n-p. Euphorbia sp.; q-s. Salvia sp.; t-u. Lopezia sp.; v-w. Poaceae; x-z. Solanum. Barra $1 \mathrm{~cm}=10 \mu \mathrm{m}$. 


\section{DISCUSIÓN}

En el estado de Morelos como en muchas partes del país, la vegetación original ha sufrido cambios por desmonte o deforestación, dedicándose amplias zonas a labores agrícolas o siendo ocupadas por vegetación secundaria (Cerros-Tlatilpa \& Espejo-Serna, 1998). Esta situación se hace evidente en las mieles analizadas en donde se determinaron tipos polínicos pertenecientes a Asteraceae, Poaceae, Lopezia, Brassica y Salvia, plantas que con frecuencia crecen a orillas de caminos, canales de riego o jardines en los alrededores de zonas urbanas.

En cuanto al número de tipos polínicos en este trabajo se determinaron 23, mientras que (Quiroz-García \& Arreguín-Sánchez, 2008) en las mieles analizadas del estado de Morelos, México, identificaron 41. En ambos trabajos el polen de la familia Asteraceae estuvo presente, lo que concuerda con lo que mencionan algunos autores (Cerros-Tlatilpa \& Espejo-Serna, 1998) de que esta familia es de las mejor representadas en diversas zonas de Morelos.

A diferencia de lo encontrado por (Quiroz-García \& Arreguín-Sánchez, 2008) en las muestras de Tlayacapan y Tepoztlán en donde se identificaron tipos polínicos propios del bosque tropical caducifolio como son Tabebuia y Bursera; en las mieles de Cuautla y Totolapan, aunque se menciona la presencia de este tipo de vegetación, no se apreció polen característico de ella, probablemente porque la primera es una localidad muy urbanizada y en Totolapan las áreas agrícolas son muy extensas.

De los tipos polínicos encontrados por (Piedras-Gutiérrez \& Quiroz-García, 2007), la familia Asteraceae, Chenopodiaceae-Amaranthaceae, Leguminosae, y los géneros Brassica, Lopezia y Salvia también fueron reportados como en este estudio, coincidiendo en que las localidades de donde se obtuvieron las muestras han sido muy modificadas por el crecimiento de la mancha urbana y el uso agrícola de los terrenos en la zona sur del Valle de México.

De acuerdo con los resultados obtenidos en este trabajo pocos tipos polínicos muestran porcentajes altos, al respecto (Cortapassi-Laurino \& Ramalho, 1988) señalan que las plantas con presencia mayor al 10\%, pueden considerarse como un recurso alimenticio importante, mientras que las especies vegetales presentes en pequeñas proporciones son consideradas recurso alternativo.

La presencia del polen de las familias Chenopodiaceae-Amaranthaceae en dos de las mieles analizadas de acuerdo con autores como (McGregor, 1981) (Kleinert-Giovannini \& ImperatrizFonseca, 1987) (Quiroz-García \& Palacios-Chávez, 1999), entre otros se debe a que son visitadas por las abejas por la gran cantidad de polen que producen y puede llegar a ser almacenado para complementar su nutrición. Además, de acuerdo con Adams \& Smith (1981) en (Castellanos-Potenciano, Ramírez-Arriaga, \& Zaldivar-Cruz, 2012) la presencia en la miel de algunas especies consideradas poliníferas se debe a que al colectar néctar las abejas, llevan polen corbicular que se mezcla con el néctar libado.

(Ordetx, Zosaya-Rubio, \& Franco-Millan, 1972) señalan que la familia Asteraceae es una familia cosmopolita, que comprende la décima parte de todas las plantas conocidas, con numerosas especies nectaríferas y poliníferas. Esto concuerda con los resultados obtenidos, ya que la familia Asteraceae se encuentra bien representada, tanto en número de especies como en frecuencia de aparición en las tres mieles, además esta familia forma parte importante de la vegetación secundaria debido a que muchas de ellas son utilizadas en la jardinería, como alimento, medicinales y muchas otras son consideradas malas hierbas; a su vez, son muy visitadas por las abejas, al presentar las inflorescencias estructuras de atracción para ellas y poseen polen pequeño lo que facilita su transporte (Cervantes, 2005).

De acuerdo con (Ordetx, Zosaya-Rubio, \& Franco-Millan, 1972), la familia Euphorbiaceae es utilizada únicamente para subsistir y en la región de estudio se encuentra con baja 
representación en las muestras analizadas, sin embargo (Proctor \& Yeo, 1973) mencionan que las abejas se inclinan por visitar con más frecuencia aquellas flores que les proporcionan néctar y polen, como es el caso de la familia Asteraceae que está presente en proporciones mayores en las mieles estudiadas.

Dentro del género Brassica, se encuentra un grupo de especies cultivadas, aunque existen también taxones como la mostaza común (B. campestris L.) que son malezas invasoras de campos de cultivo, es por ello que se halla bien representada en las tres muestras. El género Lopezia presenta flores no muy vistosas que producen polen y néctar que atraen a las abejas, es frecuente en zonas perturbadas, campos de cultivo abandonados o de forma ruderal en el bosque, en las laderas de los barrancos o cerros altos (Cervantes, 2005).

Entre los principales tipos polínicos identificados en este estudio se encontraron: Asteraceae, Brassica, Lopezia y Salvia que son considerados como buenos proveedores de néctar y polen (Ordetx, Zosaya-Rubio, \& Franco-Millan, 1972) (Villegas, 2008).

De acuerdo con los resultados obtenidos en este trabajo, el estrato mejor representado fue el herbáceo, lo que concuerda con las observaciones realizadas por (Alvarado \& Delgado, 1985) en mieles de Uxpanapa, Veracruz.

\section{CONCLUSIÓN}

Se determinaron 23 tipos polínicos pertenecientes a 18 familias, solo ocho de ellos mostraron porcentajes superiores al 10\%. Se observó que Apis mellifera en el área de estudio utiliza recursos vegetales de zonas perturbadas o relacionados con áreas de cultivo como son las Asteraceae y Brassicaceae, lo que concuerda con el estado de la vegetación de las localidades de donde proceden las mieles. La familia que mostró mayor diversidad en las muestras fue la Asteraceae con cuatro tipos polínicos. Dos de las mieles fueron caracterizadas por su origen botánico como monoflorales (Cuautla y Totolapan) y una como multifloral (Totolapan). Los recursos botánicos presentes en las mieles provienen en gran medida del estrato herbáceo al que corresponden: Arracacia, Asteraceae, Brassica, Salvia y Lopezia.

\section{LITERATURA CITADA}

Acosta-Castellanos, S., \& Palacios-Chávez, R. (2001). Plants of apicultural interest in the Pluma Hidalgo Zone, Oaxaca, México. (O. G. Clarke, Ed.) Houston, Texas, USA: American Association od Stratigraphic Palynologists.

Acosta-Castellanos, S., Quiroz-García, D. L., Arreguín-Sánchez, M. d., \& Fernández-Nava, R. (2011). Análisis polínico de tres muestras de miel de Zacatecas, México. Polibotánica(32), 179-191.

Alaniz-Gutiérrez, L., Ail-Catzim, C. E., Villanueva-Gutiérrez, R., Delgadillo-Rodríguez, J., Ortiz-Acosta, M. E., García-Moya, E., \& Medina-Cervantes, T. S. (2017). Caracterización palinológica de mieles del Valle de Mexicalli, Baja California, México. Polibotánica(43), 255-283.

Alvarado, J., \& Delgado, R. (1985). Flora apícola de Uxpanapa, Veracruz, México. Biotica, 10(3), 257-275.

Carmona, M. (1980). Contribución al conocimiento de la flora melífera del estado de Morelos. Tesis de licenciatura. Cuernavaca, Morelos, México.

Castellanos-Potenciano, B., Ramírez-Arriaga, E., \& Zaldivar-Cruz, J. M. (2012). Análisis del contenido polínico de mieles producidas por Apis mellifera L. (Hymenoptera: Apidae) en el estado de Tabasco, México. Acta Zoológica Mexicana, 28(1), 13-36. 
Cerros-Tlatilpa, R., \& Espejo-Serna, A. (1998). Contribución al estudio florístico de los cerros El sombrerito y Las Mariposas (Zoapapalotl) en el municipio de Tlayacapan, Morelos, México. Polibótanica(8), 29-46.

Cervantes, H. (2005). Análisis polínico de la miel de Ixmiquilpan, Hidalgo. Tesina de licenciatura. Escuela Nacional de Ciencias Biológicas-IPN.

Contreras-Escareño, F., Pérez-Armendáriz, B., Echazarreta, C. M., Cavazos-Arroyo, J., MacíasMacías, J. O., \& Tapia-González, J. M. (2013). Características y situación actual de la apicultura en las regiones sur y sureste de Jalisco, México. Revista Mexicana de Ciencias Pecuarias, 4(3), 387-398.

Córdova-Córdova, C., Ramírez-Arriaga, E., Martínez-Hernández, E., \& Zaldivar-Cruz, J. (2013). Caracterización botánica de miel de abeja (Apis mellifera) de cuatro regiones del estado de Tabasco, México, mediante técnicas melisopalinológicas. Universidad y Ciencia Trópico Húmedo, 29(1), 163-178.

Cortapassi-Laurino, M., \& Ramalho, M. (1988). Pollen harvest by africanizad Apis mellifera and Trigona spinipes in Sao Paulo. Botanical and Ecological reviews. Apidologie, 10(1), 1-24.

Erdtman, G. (1952). Pollen Morphology and Plant Taxonomy. Angiosperms: An Introduction to Palynology (Vol. I). Waltham, Mass.: Chronica Botanica.

Kleinert-Giovannini, A., \& Imperatriz-Fonseca, V. L. (1987). Aspects of the tropic noche of Melipona marginata marginata Lepeletier (Apidae, Meliponinae). Apidologie, 18(1), 68-100.

Lieux, M. (1972). A melissopalynological study of 54 Louisiana (USA) honeys. Review of Paleobotany and Palynology(13), 95-124.

Lieux, M. (1980). Acetolysis applied to microscopical honey analysis. Grana(19), 57-61.

Loveaux, J., Mauricio, A., \& Vorwohl, G. (1978). Methods of melissopalynology. Bee World, 59(4), 139-157.

McGregor, S. (1981). La apicultura en los Estados Unidos. Distrito Federal: Limusa.

Ordetx, G., Zosaya-Rubio, J. A., \& Franco-Millan, W. (1972). Estudio de la flora apicola nacional. Folleto misceláneo(2), 1-94. Chapingo, Estado de México, México: Dirección General de Extensión Apícola.

Palacios, C. (1968). Morfología de los granos de polen de árboles del estado de Morelos. Anales de la Escuela Nacional de Ciencias Biológicas(16), 41-93.

Palacios, C., Ludlow-Wiechers, B., \& Villanueva, R. (1991). Flora palinológica de la reserva de la Biosfera de Sian Ka'an, Quintana Roo, México. Chetumal, Quintana Roo: Centro de Investigaciones de Quintana Roo.

Piedras-Gutiérrez, B., \& Quiroz-García, D. (2007). Estudio melisopalinológico de dos mieles de la porción sur del Valle de México. Polibotánica(23), 57-75.

Porter, L. (2001). Landscape ecology of apiculture in the maya area of La Montaña. Tesis de doctorado, 196 pp. Campeche, México: Universidad de Florida.

Proctor, M., \& Yeo, P. (1973). The pollination of flowers. (G. W. Sons, Ed.)

Quiroz-García, D., \& Arreguín-Sánchez, M.L. (2008). Determinación palinológica de los recursos florales utilizados por Apis mellifera L. (Hymenoptera: Apidae) en el estado de Morelos, México. Polibotánica(26), 159-173.

Quiroz-García, D., \& Palacios-Chávez, R. (1999). Determinación palinológica de los recursos florales utilizados por Centris inermes Friese (Hymenoptera: Apidae) en Chamela, Jalisco, México. Polibotánica(10), 59-72.

Ramalho, M., Imperatriz-Fonseca, V. L., Kleinert-Giovannini, A., \& Cortopassi Laurino, M. (1985). Exploitation of floral resources by Plebeia remota Holmberg (Apidae, Meliponinae). Apidologie, 16(3), 307-330.

Ramírez, R., \& Montenegro, G. (2004). Certificación del origen botánico de miel y polen corbicular pertenecientes a la comuna de Litueche, IV Región de Chile. Cienc. Inv. Agr., 31(3), 197-211.

Roubik, W., \& Moreno, J. E. (1991). Pollen and spores of Barro Colorado Island. Monographs in systematic botany from the Missouri Botanical Garden, 36, 1-268. 


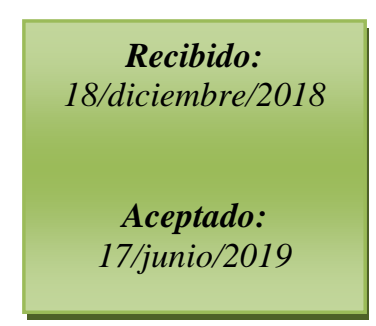

Vélez, I., Espinoza, G. J., Amaro, G., \& Arechavaleta, V. M. (2016). Tipología y caracterización de apicultores del estado de Morelos, México. Revista Mexicana de Ciencias Pecuarias, 7(4), 507-524.

Villegas, P. (2008). Plantas de importancia apícola registradas en México. Tesina de licenciatura. Distrito Federal, México: Escuela Nacional de Ciencias Biológicas-IPN. Obtenido de http://www.e- 